\title{
Importance of anisotropy in the evaluation of dispersion interactions
}

\author{
A. Krishtal, ${ }^{1}$ K. Vannomeslaeghe, ${ }^{2}$ D. Geldof, ${ }^{1}$ C. Van Alsenoy, ${ }^{1}$ and P. Geerlings ${ }^{3}$ \\ ${ }^{1}$ Department of Chemistry, University of Antwerp, Universiteitsplein 1, B-2610 Antwerp, Belgium \\ ${ }^{2}$ Department of Pharmaceutical Sciences, University of Maryland School of Pharmacy, 20 North Pine Street, HSF II-629, Baltimore, \\ Maryland 21201, USA \\ ${ }^{3}$ Algemene Chemie, Vrije Universiteit Brussel, Pleinlaan 2, B-1050 Brussels, Belgium \\ (Received 21 October 2010; published 14 February 2011)
}

\begin{abstract}
The evaluation of dispersion interaction energies, with the goal of correcting the performance of density functional theory (DFT) methods, is currently a topic of intensive research. Most of the dispersion-corrected DFT methods (DFT-D) rely on an additive correction expression based on the use of isotropic dispersion coefficients. This, however, undermines an important aspect of the interaction, i.e., its anisotropic nature. We demonstrate that, for systems of sufficient size, such as benzene dimers and DNA base pairs, the inclusion of anisotropy, through the use of the Hirshfeld method, results in an increase of dispersion energy values by up to $30 \%$.
\end{abstract}

DOI: 10.1103/PhysRevA.83.024501

PACS number(s): 31.15.es

The growth of computational capacity over the recent decade has widened the application window of quantum chemical methods to systems of biochemical interest. In particular, density functional theory (DFT) [1,2] has proved to be a popular choice for this sort of application. The expansive range of data thus produced has, however, brought forth not only the merits of the broad spectrum of available functionals but also their flaws. A major drawback, for example, is their lack of consistency when it comes to describing dispersion interactions [3]. The abundance of stacking interactions in biochemically active systems requires an immediate, accurate, and not overly computationally demanding solution for this problem if we are to continue applying DFT to these systems. As one could expect, many suggestions have been made to correct DFT energies for dispersion interactions [4-20]. A great many of these methods rely on an additive correction scheme, which is rooted in a perturbation theory model for long-range interactions [21-24]. However, an important aspect of this model has been left out by most of these schemes, namely, the anisotropic nature of dispersion. In this work, we propose a method to include anisotropy in a straightforward fashion and demonstrate its impact on a limited yet illustrative set of systems.

The perturbation theory treatment of dispersion [21-24] describes the interaction of two systems $A$ and $B$ at a sufficiently large distance $R_{A B}$ as the potential energy of the charge distribution of system $A$ in the external field created by system $B$. Due to the large $R_{A B}$ condition, the potential $\phi_{B}\left(r_{i}\right)$ can be expanded in a Taylor series and subsequently replaced by a multipolar expansion, which leads to the following form of the perturbation Hamiltonian:

$$
\begin{aligned}
V_{A B}= & T_{B} q_{B} q_{A}+\sum_{\alpha} T_{B_{\alpha}}\left(q_{A} \mu_{B_{\alpha}}-q_{B} \mu_{A_{\alpha}}\right) \\
& +\sum_{\alpha \beta} T_{\alpha \beta}\left(\frac{1}{3} q_{A} \Theta_{B_{\alpha \beta}}+\frac{1}{3} q_{B} \Theta_{A_{\alpha \beta}}-\mu_{A_{\alpha}} \mu_{B_{\beta}}\right),
\end{aligned}
$$

where $\alpha, \beta, \gamma$, and $\delta$ represent the Cartesian directions and $q$, $\mu, \Theta$, and $\Omega$ stand for the charges, dipole moments, quadrupole moments, and octopole moments, respectively. The $T$ tensors depend on $R_{A B}$ and its derivatives: $T_{B}=R_{A B}^{-1}, T_{B_{\alpha}}=\nabla_{\alpha} R_{A B}^{-1}$, and so forth. The dispersion energy contribution that emerges from this perturbation Hamiltonian is given by

$$
E_{\mathrm{disp}}=-\sum_{j_{A} \neq n_{A}} \sum_{j_{B} \neq n_{B}} \frac{\left|\left\langle\psi_{n_{A}}^{(0)} \psi_{n_{B}}^{(0)}\left|V_{A B}\right| \psi_{j_{A}}^{(0)} \psi_{j_{B}}^{(0)}\right\rangle\right|^{2}}{\left(W_{j_{A}}^{(0)}-W_{n_{A}}^{(0)}\right)+\left(W_{j_{B}}^{(0)}-W_{n_{B}}^{(0)}\right)},
$$

where $n_{A}$ and $n_{B}$ stand for ground states, $j_{A}$ and $j_{B}$ stand for the excited states, $\psi_{A}^{(0)}$ and $\psi_{B}^{(0)}$ are the wave functions, and $W_{A}^{(0)}$ and $W_{B}^{(0)}$ are the eigenvalues of the unperturbed systems $A$ and $B$. In evaluating Eq. (2), two major approximations are usually made: First, the sum over $\Delta W_{0}$ in the denominator is approximated by a "mean excitation energy," and second, the perturbation Hamiltonian is spherically averaged, yielding an effective potential depending only on the interatomic distance $R_{A B}$. The following familiar isotropic expression then results:

$$
E_{\mathrm{disp(iso)}}^{A B}=-\left(\frac{C_{6}}{R_{A B}^{6}}+\frac{C_{8}}{R_{A B}^{8}}+\frac{C_{10}}{R_{A B}^{10}}+\cdots\right),
$$

which is used in most of the currently developed dispersioncorrected DFT methods (DFT-D), although the exact expression used for the isotropic dispersion coefficients varies significantly, depending on the sophistication level of the method. It must be mentioned that in some works the first approximation is omitted [5,13,14,25-28], although Osinga et al. [26] have even kept the anisotropic expression by evaluating the $1 / R^{n}$ expressions through spherical harmonics with Racah's normalization. The increase in the computational expense of this method is such that its application to systems of extended size becomes prohibitive. In a more recent work, Sato and Nakai [27,28] employ a local response approximation that allows the calculation of dispersion coefficients in terms of frequency-dependent polarizabilities in a more simplified fashion. Also, in this method, spherical harmonics are used to account for the anisotropic character.

In this Brief Report we suggest a method for employing the first approximation while retaining the anisotropic nature of the perturbation Hamiltonian in Eq. (1), which will prove to be essential in evaluating the correct dispersion energies in larger systems. Following Buckingham in approximating the 
denominator in Eq. (2), one obtains the following expressions for the first three terms of the series [22-24]:

$$
\begin{aligned}
E_{\mathrm{disp}}\left(R^{-6}\right)= & -\frac{1}{4} \frac{U_{A} U_{B}}{U_{A}+U_{B}} \operatorname{tr}\left[\mathbf{T}_{2} \boldsymbol{\alpha}_{B} \mathbf{T}_{2} \boldsymbol{\alpha}_{A}^{\dagger}\right] \\
E_{\mathrm{disp}}\left(R^{-7}\right)= & -\frac{1}{4} \frac{U_{A} U_{B}}{U_{A}+U_{B}}\left(\frac{2}{3} \operatorname{tr}\left[\mathbf{T}_{2} \boldsymbol{\alpha}_{A} \mathbf{T}_{3} \mathbf{A}_{B}^{\dagger}\right]\right. \\
& \left.-\frac{2}{3} \operatorname{tr}\left[\mathbf{T}_{2} \boldsymbol{\alpha}_{B} \mathbf{T}_{3} \mathbf{A}_{A}^{\dagger}\right]\right) \\
E_{\mathrm{disp}}\left(R^{-8}\right)=- & \frac{1}{4} \frac{U_{A} U_{B}}{U_{A}+U_{B}}\left(\frac{1}{3} \operatorname{tr}\left[\boldsymbol{\alpha}_{A} \mathbf{T}_{3} \mathbf{C}_{B}^{\dagger} \mathbf{T}_{3}^{\dagger}\right]\right. \\
+ & \frac{1}{3} \operatorname{tr}\left[\boldsymbol{\alpha}_{B} \mathbf{T}_{3} \mathbf{C}_{A}^{\dagger} \mathbf{T}_{3}^{\dagger}\right]-\frac{2}{9} \operatorname{tr}\left[\mathbf{T}_{3}\left(\mathbf{A}_{A} \mathbf{T}_{3}^{\dagger} \mathbf{A}_{B}\right)^{\dagger}\right] \\
& \left.-\frac{2}{9} \operatorname{tr}\left[\mathbf{T}_{2} \mathbf{A}_{A} \mathbf{T}_{4} \mathbf{A}_{B}^{\dagger}\right]\right)
\end{aligned}
$$

where $U$ are the above-mentioned "mean excitation energies." $\boldsymbol{\alpha}, \mathbf{A}$, and $\mathbf{C}$ are the static dipole polarizability, quadrupoledipole polarizability, and quadrupole polarizability tensors. Here the $\mathbf{A}$ tensor is built as a $3 \times 9$ tensor, where the rows represent the three dipole components and the columns represent the nine quadrupole components. Analogously, $\mathbf{C}$ is a $9 \times 9$ tensor. The quadrupole-dipole polarizability tensor of a spherically symmetric system is zero; hence, the isotropic counterpart of this property $A^{\text {iso }}=0$. The tensor values of the $\mathbf{C}$ tensor of a spherical symmetric system are given by $\mathbf{C}_{\alpha \beta, \gamma \delta}=C^{\text {iso }}\left[\frac{1}{2}\left(\delta_{\alpha \gamma} \delta_{\beta \delta}+\delta_{\alpha \delta} \delta_{\beta \gamma}\right)-\frac{1}{3} \delta_{\alpha \beta} \delta_{\gamma \delta}\right]$, where $C^{\text {iso }}$ is defined as $\frac{1}{5} \operatorname{tr} \mathbf{C}$. $\mathbf{T}_{2}, \mathbf{T}_{3}$, and $\mathbf{T}_{4}$ are $3 \times 3,3 \times 9$, and $9 \times 9$ tensors that contain the elements of $T_{B_{\alpha \beta}}, T_{B_{\alpha, \beta \gamma}}$, and $T_{B_{\alpha \beta, \gamma \delta}}$, respectively. In the isotropic case, one can replace the tensors $\boldsymbol{\alpha}, \mathbf{A}$, and $\mathbf{C}$ with their isotropic counterparts, and since $\operatorname{tr}\left[\mathbf{T}_{2} \mathbf{T}_{2}\right]=\frac{6}{R^{6}}$ and $\operatorname{tr}\left[\mathbf{T}_{3} \mathbf{T}_{3}^{\dagger}\right]=\frac{90}{R^{8}}$, these equations reduce to

$$
\begin{gathered}
E_{\text {disp }}^{\text {iso }}\left(R^{-6}\right)=-\frac{3}{2} \frac{U_{A} U_{B}}{U_{A}+U_{B}} \frac{1}{R^{6}} \alpha_{A}^{\text {iso }} \alpha_{B}^{\text {iso }}, \\
E_{\text {disp }}^{\text {iso }}\left(R^{-8}\right)=-\frac{3}{2} \frac{U_{A} U_{B}}{U_{A}+U_{B}} \frac{1}{R^{8}} 5\left(\alpha_{A}^{\text {iso }} C_{B}^{\text {iso }}+\alpha_{B}^{\text {iso }} C_{A}^{\text {iso }}\right),
\end{gathered}
$$

while $E_{\text {disp }}^{\text {iso }}\left(R^{-7}\right)=0$. The approximation of the denominator in Eq. (2) is valid when the mean excitation energies $U$ are similar to $\left(W_{j}^{(0)}-W_{n}^{(0)}\right)$. For systems $A$ and $B$, which consist of multiple atoms $a$ and $b$, each of the dispersion correction terms in Eqs. (4)-(6) can be approximated as a sum over all pairwise contributions [29]: $E_{\text {disp }}=\sum_{a \in A} \sum_{b \in B} E_{\text {disp }}^{a b}$. As a consequence, all quantities with subscripts $A$ and $B$ in Eqs. (4)-(6) need to be evaluated for atoms $a$ and $b$. Their evaluation requires, however, the knowledge of atomic polarizability tensors, which can be obtained by means of the Hirshfeld method [30,31].

The Hirshfeld method allows us to partition the molecular density into a sum of atomic contributions by means of a set of normalized atomic weight functions $\omega_{a}(r)$, which are constructed from the densities of the free spherically symmetric atoms, normalized by the sum of all the atomic densities, which is also referred to as the "promolecule." In the improved, iterative version of the Hirshfeld method [31] used throughout this work, the weight function is determined iteratively from atomic densities obtained in the previous iteration until convergence is achieved.
In the framework of the Hirshfeld method, the $\alpha \beta$ element of the intrinsic atomic polarizability tensor is defined as [32]

$$
\boldsymbol{\alpha}_{\alpha \beta}^{a}=-\int\left(\alpha-a_{\alpha}\right) \omega_{a}(r) \rho^{(\beta)}(r) d \vec{r},
$$

where $a_{\alpha}$ stands for the $\alpha$ coordinate of atom $a$ and $\rho^{(\beta)}$ is the first-order molecular density perturbed by a static dipole field in the Cartesian $\beta$ direction. The elements of the total molecular polarizability tensor can be reconstructed exactly by summing over all the intrinsic polarizabilities of the atoms in the molecule and their respective charge delocalization contributions [32]:

$$
\boldsymbol{\alpha}_{\alpha \beta}=\sum_{a} \boldsymbol{\alpha}_{\alpha \beta}^{a}+a_{\alpha} q_{a}^{(\beta)},
$$

where $q_{a}^{(\beta)}=-\int \omega_{a}(r) \rho^{(\beta)}(r) d \vec{r}$ is the first-order perturbed atomic charge. We have validated the Hirshfeld method for partitioning dipole polarizabilities on a broad range of systems [32-35], including the evaluation of the isotropic dispersion coefficients and the anisotropic $C_{6}$ coefficient $[9,12]$. In order to evaluate the anisotropic $C_{7}$ and $C_{8}$ coefficients, we now introduce the definitions of the intrinsic quadrupole-dipole and quadrupole polarizability tensors:

$$
\mathbf{A}_{\gamma, \alpha \beta}^{a}=-\int\left(\alpha-a_{\alpha}\right)\left(\beta-a_{\beta}\right) \omega_{a}(r) \rho^{(\gamma)}(r) d \vec{r}
$$

and

$$
\mathbf{C}_{\alpha \beta, \gamma \delta}^{a}=-\int\left(\alpha-a_{\alpha}\right)\left(\beta-a_{\beta}\right) \omega_{a}(r) \rho^{(\gamma \delta)}(r) d \vec{r},
$$

where $\rho^{(\gamma \delta)}(r)$ is the first-order molecular density, perturbed by the quadrupole field in the $\gamma \delta$ direction. Finally, the Hirshfeld method is also used in the evaluation of the mean excitation energies $U_{a}$, where we follow Becke and Johnson's proposal [29,36] that $U_{a}=\left\langle\mu_{a}^{2}\right\rangle / 3 \alpha_{a}^{\text {iso }}$, with $\left\langle\mu_{a}^{2}\right\rangle$ being the expectation value of the square of the atomic dipole moment and $\alpha_{a}^{\text {iso }}$ being the isotropic value of the intrinsic atomic polarizability.

The origin of the equations in perturbation theory has an important impact on their validity when applied to any system $A B$ : the assumption of the large distance $R_{A B}$ allowed the expansion of the potential $\phi_{B}$ as a Taylor series and its subsequent expansion as a multipole series. This assumes a purely electrostatic interaction, without any significant electron exchange. When applying these equations on systems where $R_{A B}$ is not sufficiently large for those two conditions to be valid, a damping function must be used. However, in order to demonstrate the effect of anisotropy on the values of dispersion energies, we are interested in displaying values that are unmasked and unaltered by damping. Therefore, we will limit our discussion to only a set of stacking benzene dimers and nucleic base pairs, where the distance between any two atoms between the monomers is sufficiently large.

Our choice of systems includes the three well-studied isomers of the benzene dimer, namely, the $C_{2 h}$ parallel-displaced dimer [Fig. 1(a)], the $C_{2 v}$ T-shaped dimer [Fig. 1(b)], and the $D_{6 h}$ [Fig. 1(c)] sandwich dimer. Also, due to the importance of anisotropy in biochemically active systems, we will examine two nucleic base pairs in a stacking configuration, namely, adenine-thymine (AT) and uracil-uracil (UU). The geometries of the nucleic base pairs were obtained from the SS2 


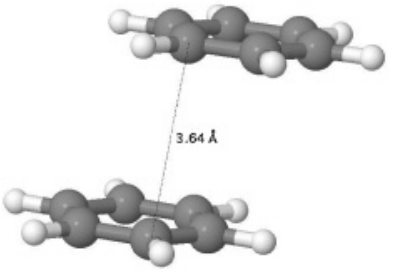

(a)

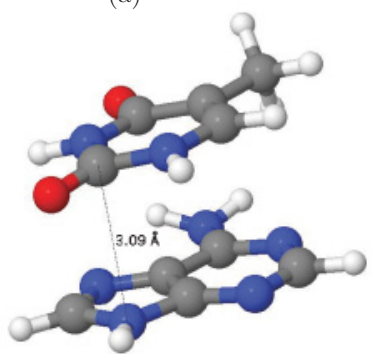

(d)

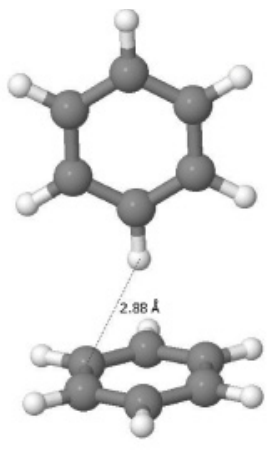

(b)

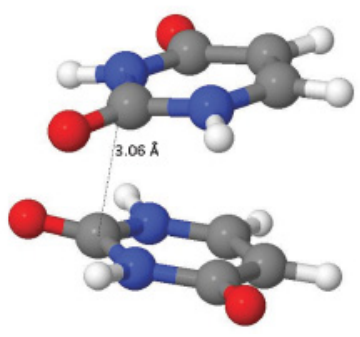

(e)

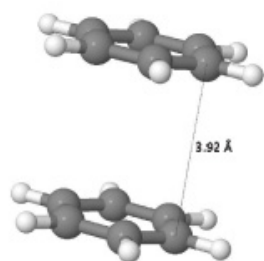

(c)
FIG. 1. (Color online) The five stacking dimers used in this work. (a) The $C_{2 h}$ paralleldisplaced benzene dimer. (b)The $C_{2 v}$ T-shaped benzene dimer. (c) The $D_{6 h}$ sandwich benzene dimer. (d) The adenine-thymine dimer. (e) The uracil-uracil dimer. benchmark data set as compiled by Jurecka et al. [37], and the geometries of the benzene dimers were obtained from Janowski and Pulay [38] as compiled by Jurecka et al. [37]. The calculations of the global polarizabilities of the monomers have been performed using GAUSSIAN03 [39] and partitioned using the STOCK program [40]. Table I lists the dispersion energies calculated for these dimers using the anisotropic model [Eqs. (4)-(6)] and the isotropic model [Eqs. (7) and (8)]. For each model, we include the contribution from the $R^{-6}$ term to illustrate the convergence behavior of the series. Finally, in order to demonstrate the dependence of the dispersion energy correction on the functional, we present results calculated with three functionals with different natures, namely, Becke three-parameter Lee-Yang-Parr (B3LYP) [41], Perdew-Burke-Ernzerhof (PBE) [42], and
Tao-Perdew-Staroverov-Scuseria (TPSS) [43], each combined with the $6-311++G^{* *}$ basis set [44].

Three main conclusions can be drawn from the results in Table I. First, the influence of the choice of the functional is limited for all systems, which demonstrates the robust character of the method.

Second, the addition of the $E_{\text {disp }}\left(R^{-7}\right)$ and $E_{\text {disp }}\left(R^{-8}\right)$ terms increases the dispersion energy by approximately $50 \%$, illustrating that the series has not yet converged and higher terms, namely, $E_{\text {disp }}\left(R^{-9}\right)$ and $E_{\text {disp }}\left(R^{-10}\right)$, should be taken into account. Finally and most importantly, the contribution of anisotropy to the dispersion energy of these two systems is very significant, where it accounts for an average of $25 \%$ of the value for the $E_{\text {disp }}\left(R^{-6}\right)$ term and $20 \%$ of the value for the total sum of the three terms. This finding illustrates

TABLE I. The dispersion energy corrections (in kcal/mol) of the $C_{2 h}$ parallel-displaced benzene dimer, the $D_{6 h}$ sandwich benzene dimer, the $C_{2 v}$ T-shaped benzene dimer, the adenine-thymine dimer, and the uracil-uracil dimer, calculated using the anisotropic [Eqs. (4)-(6)] and isotropic [Eqs. (7) and (8)] models.

\begin{tabular}{|c|c|c|c|c|c|c|c|}
\hline Dimer & Functional & $E_{\text {disp }}^{\text {iso }}\left(R^{-6}\right)$ & $E_{\text {disp }}\left(R^{-6}\right)$ & $\Delta E$ & $E_{\text {disp }}^{\text {iso }}$ & $E_{\text {disp }}$ & $\Delta E$ \\
\hline \multirow[t]{3}{*}{$\left(C_{6} H_{6}\right)_{2}-C_{2 h}$} & B3LYP & -1.37 & -1.88 & -0.51 & -2.53 & -3.21 & -0.68 \\
\hline & PBE & -1.39 & -1.88 & -0.49 & -2.58 & -3.23 & -0.65 \\
\hline & TPSS & -1.35 & -1.84 & -0.49 & -2.47 & -3.12 & -0.65 \\
\hline \multirow[t]{3}{*}{$\left(C_{6} H_{6}\right)_{2}-D_{6 h}$} & B3LYP & -1.01 & -1.47 & -0.46 & -1.76 & -2.72 & -0.96 \\
\hline & PBE & -1.02 & -1.47 & -0.44 & -1.79 & -2.72 & -0.93 \\
\hline & TPSS & -1.00 & -1.44 & -0.44 & -1.72 & -2.63 & -0.91 \\
\hline \multirow[t]{3}{*}{$\left(C_{6} H_{6}\right)_{2}-C_{2 v}$} & B3LYP & -1.14 & -1.33 & -0.19 & -2.28 & -2.57 & -0.29 \\
\hline & PBE & -1.15 & -1.35 & -0.20 & -2.33 & -2.61 & -0.28 \\
\hline & TPSS & -1.12 & -1.32 & -0.20 & -2.24 & -2.52 & -0.28 \\
\hline \multirow[t]{3}{*}{ Adenine-thymine } & B3LYP & -3.17 & -4.24 & -1.07 & -6.30 & -7.77 & -1.47 \\
\hline & PBE & -3.30 & -4.35 & -1.06 & -6.64 & -8.08 & -1.44 \\
\hline & TPSS & -3.21 & -4.26 & -1.05 & -6.39 & -7.82 & -1.43 \\
\hline \multirow[t]{3}{*}{ Uracil-uracil } & B3LYP & -2.26 & -3.09 & -0.83 & -4.28 & -5.29 & -1.01 \\
\hline & PBE & -2.37 & -3.18 & -0.81 & -4.55 & -5.53 & -0.97 \\
\hline & TPSS & -2.31 & -3.11 & -0.81 & -5.05 & -6.01 & -0.97 \\
\hline
\end{tabular}


that the approximation of the spherical symmetrization of the perturbation Hamiltonian is not one to be made lightly. This remarkable influence of anisotropy has gone unnoticed until now due to the small size of the systems that are traditionally used as a test set, where the distortion of the symmetry is limited [26]. However, if one applies this model on systems of sufficient size, as was done in this study, the assumption of isotropic Hamiltonian becomes invalid. Finally, we note that there is no trivial correlation between the total symmetry of the complex and the effect of anisotropy in this particular data set. The largest influence of the anisotropy can be seen for the $D_{6 h}$ sandwich benzene dimer, where the absolute values are increased by $35 \%$, while the dispersion energy of the $C_{2 v}$ T-shaped benzene dimer is influenced the least by anisotropy, with an increase of $10 \%$. This increment in values improves the overall performance of the method, although the total dispersion energies are still underestimated. As stated above, additional higher-term energies are needed to reproduce the dispersion interaction energies to the full extent.
In conclusion, we have shown that the inclusion of anisotropy in the model increases the resulting dispersion energy substantially. The inclusion of anisotropy in the model is present on three levels: first, through the use of the anisotropic expression that contains additional terms involving dipole-quadrupole polarizablities, which vanish in the isotropic case, second, through the use of the $T$ tensors, which contain information on the orientation of the atoms with respect to each other, and, finally, through the use of atomic polarizability tensors instead of isotropic polarizabilities. Based on these results we intend to develop our anisotropic DFT-D method further to include higher-term energies, which are clearly needed to reproduce accurate dispersion energies.

The authors are thankful to the University of Antwerp for access to the university's CalcUA Supercomputer Cluster. A.K. acknowledges Research Foundation Flanders (FWO) for financial support.
[1] P. Hohenberg and W. Kohn, Phys. Rev. 136, B864 (1964).

[2] R. G. Parr and W. Yang, Density Functional Theory of Atoms and Molecules (Oxford University Press, New York, 1989).

[3] S. Kristyán and P. Pulay, Chem. Phys. Lett. 229, 175 (1994).

[4] S. Grimme, J. Comput. Chem. 25, 1463 (2004).

[5] I. Adamovic and M. S. Gordon, Mol. Phys. 103, 379 (2005).

[6] A. Heßelmann, G. Jansen, and M. Schütz, J. Chem. Phys. 122, 014103 (2005).

[7] H.-Y. Kim et al., J. Chem. Phys. 124, 074504 (2006).

[8] J. F. Dobson, A. White, and A. Rubio, Phys. Rev. Lett. 96, 073201 (2006).

[9] A. Olasz et al., J. Chem. Phys. 127, 224105 (2007).

[10] A. Banerjee, J. Autschbach, and A. Chakrabarti, Phys. Rev. A 78, 032704 (2008).

[11] A. White and J. F. Dobson, Phys. Rev. B 77, 075436 (2008).

[12] A. Krishtal et al., J. Chem. Phys. 130, 174101 (2009).

[13] A. Tkatchenko and M. Scheffler, Phys. Rev. Lett. 102, 073005 (2009).

[14] H. V. Nguyen and S. de Gironcoli, Phys. Rev. B 79, 115105 (2009).

[15] F. O. Kannemann and A. D. Becke, J. Chem. Theory Comput. 6, 1081 (2010).

[16] M. Zgarbová et al., PhysChemChemPhys 12, 10476 (2010).

[17] S. N. Steinmann and C. Corminboeuf, J. Chem. Theory Comput. 6, 1990 (2010).

[18] E. R. Johnson et al., J. Am. Chem. Soc. 132, 6498 (2010).

[19] Y. Liu and W. A. Goddard III, J. Phys. Chem. Lett. 1, 2550 (2010).

[20] A. J. Misquitta, J. Spencer, A. J. Stone, and A. Alavi, Phys. Rev. B 82, 075312 (2010).

[21] F. London, Trans. Faraday Soc. 33, 8 (1937).

[22] A. D. Buckingham, Q. Rev. (London) 13, 189 (1959).

[23] A. D. Buckingham, Adv. Chem. Phys. 12, 107 (1967).
[24] A. D. Buckingham, in Intermolecular Interactions: From Diatomics to Biopolymers (Wiley, Bath, 1978).

[25] A. Heßelmann and G. Jansen, Chem. Phys. Lett. 367, 778 (2003).

[26] V. P. Osinga et al., J. Chem. Phys. 106, 5091 (1997).

[27] T. Sato and H. Nakai, J. Chem. Phys. 131, 224104 (2009).

[28] T. Sato and H. Nakai, J. Chem. Phys. 133, 194101 (2010).

[29] A. D. Becke and E. R. Johnson, J. Chem. Phys. 124, 014104 (2006).

[30] F. L. Hirshfeld, Theor. Chim. Acta 44, 129 (1977).

[31] P. Bultinck et al., J. Chem. Phys. 126, 144111 (2007).

[32] A. Krishtal et al., J. Chem. Phys. 125, 034312 (2006).

[33] A. Krishtal, P. Senet, and C. Van Alsenoy, J. Chem. Theory Comput. 4, 426 (2008).

[34] A. Krishtal, P. Senet, and C. Van Alsenoy, J. Chem. Theory Comput. 4, 2122 (2008).

[35] A. Krishtal, P. Senet, and C. Van Alsenoy, J. Chem. Phys. 133, 154310 (2010).

[36] P. W. Atkins and R. S. Friedman, Molecular Quantum Mechanics, 3rd ed. (Oxford University Press, New York, 1997).

[37] P. Jurecka et al., PhysChemChemPhys 8, 1985 (2006).

[38] T. Janowski and P. Pulay, Chem. Phys. Lett. 447, 27 (2007).

[39] M. J. Frisch et al., GAUSSIAN 03, revision C.02, Gaussian, Inc., Wallingford, CT, 2004.

[40] B. Rousseau, A. Peeters, and C. Van Alsenoy, Chem. Phys. Lett. 324, 189 (2002).

[41] P. J. Stephens et al., J. Phys. Chem. 98, 11623 (1994).

[42] J. P. Perdew, K. Burke, and M. Ernzerhof, Phys. Rev. Lett. 78, 1396 (1997).

[43] J. M. Tao, J. P. Perdew, V. N. Staroverov, and G. E. Scuseria, Phys. Rev. Lett. 91, 146401 (2003).

[44] A. D. McLean and G. S. Chandler, J. Chem. Phys. 72, 5639 (1980); K. Raghavachari et al., ibid. 72, 650 (1980). 